\title{
Optimal Configuration PV System Grid Connection based on the Minimum Transmission Loss
}

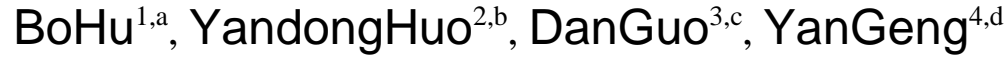 \\ ${ }^{1.3 .4}$ Shenyang Agricultural University, Shenyang, 110866,China \\ ${ }^{2}$ The State Grid Liaoning Province Power Supply Company Liaoning, Fuxin 123200, China

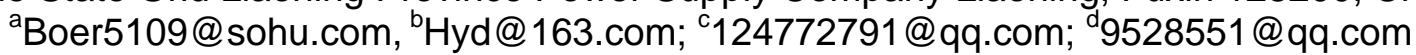

Keywords:PV grid connection; Transmission loss; Binary search algorithm; SPSS analysis; Linear regression analysis; Flow distribution; Distribution system reconstruction.

Abstract. This paper integrates the binary search algorithm and the load flow calculation to find the position and the injection capacity of the PV grid-connected injection node, it bases on minimizing the transmission loss and enhancing the voltage of the end. As verified by the IEEE33 node distribution system, the simulation resultshows that with such method, the transmission loss dropsby $67.7 \%$, the voltage of end node is improvedby $7.6 \%$ on average, so that it has a good effect on reduction of the transmission loss as well as improvement of the end voltage. From the SPSS varianceanalysis with the node parameter of the IEEE33 as the sample, this paper obtains the analysis of the correlation between the PV injection mode and the node voltage of the distribution network. It proposes the linear regression equation for PV grid-connected node voltage and the PV injection mode, where the prediction model has a good fitting effect so that it can achieve a good interaction between the main grid and the PV system; in addition, it selects the end node of the power distribution system as a small sample for T-test comparison and analysis; thus, it obtains the conclusion of the significance of the impact of PV nodes, indicates the priority of the PV injection node, puts forward the rationalization proposals for PV grid connection, and also provides the theory support for the reconstruction of the PV distribution system in the future.

\section{Introduction}

Due to its intermittent and discontinuity, the photovoltaic power generation system may reduce the operation reliability of the main power grid; however, since the distributed photovoltaic renewable energy represented by photovoltaic power generation grows extremely fast on a world scale, photovoltaic power generation's dominant status in the energy will be an inevitable trend of development ${ }^{[1-3]}$; with the increasing proportion of photovoltaic power generation in the distribution network, its operation would affect the voltage of the connected point and the load flow distribution of the distribution network, while its instability will be result in fluctuations in the voltage of the distribution network. Controllable and adjustable PV grid-connected power generation is the problem to be solved in the distribution system. We can see that studies on PV grid connection at home and abroad focus on analysis of voltage at grid-connected points ${ }^{[4-6]}$. So the key is to make reasonable addressing and constantvolume for PV grid connection.

This paper combined the forward-back substitution method and the Newton-Ralfson load flow calculation method where binary search algorithm was added, and proposed the PV grid-connected addressing and sizing method to reasonably complete PV system and network optimization with the objective of minimum active power loss and by aiming at enhancing the end voltage at the lowpressure side. From the simulation verification of the IEEE33 node distribution system, the method reduces the loss significantly and enhances the end voltage greatly.

This paper conducted the SPSS variance analysis with the node parameter of the IEEE33 as the sample, and proposed the linear regression equation for PV grid connected node voltage and injection mode based on the significance of the correlation analysis of the PV injection mode and the node voltage of the distribution network; in addition, it selects the end node of the power distribution system as a small sample for T-test comparison and analysis; thus, it obtains the conclusion thatthe PV injection node has significant impact on the node voltage. Based on 
theanalysis results, it identified the priority of the PV injection node for selectivePV grid-connected operation in the distribution network,put forward the rationalization proposals for PV grid connection, and also provided the theory support for the reconstruction of the PV distribution system inthe future.

\section{Addressing and Sizing of PV System into the Power Distribution Network}

The IEEE 33 node distribution system is shown in Figure 1. This paper calculatedeach branch power, the first branch power and each node voltage with the initial voltage and the injection power as the known conditions by combining theload flow calculation of the distribution system based on the forward-back substitution method and the Newton- Ralfson method and using the first iteration result of the forward-back substitution method as the of the initial value of the Newton-Ralfson method. On this basis, with the binary search algorithm, find the PV injection node from the last terminal sub-feeder (8-33), For sub-feeders(6-16), (4-25) and(3-22), find the photovoltaic injection node according to the same method, which are nodes 11,24 and18 respectively and are connected to the $800 \mathrm{KW}, 1019 \mathrm{KW}$ and $752 \mathrm{KW}$ PV system. Theequivalentmulti-node diagram after PV injection is shown in Fig.2.
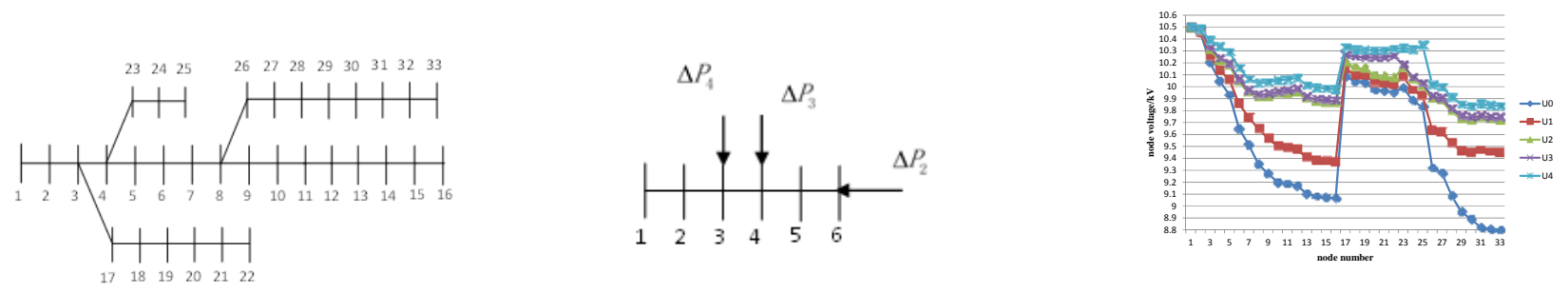

Fig.1IEEE 33 Node Power Distribution SystemFig.2 Equivalent Node Diagram Distribution Fig.3 Voltage Change Curve of PV Grid-Connected System Network after PV Injection

The effect of the PV system as a "special load" into the grid on thedistribution network and the node voltage changes are shown in Fig. 3. The end node $(33,25,22$ and 16) voltage is increased by $7.6 \%$ on average, while the voltage of the end node33 is increased by to $12.1 \%$ at maximum, where the end node33s and the node16 play the most significant role. As shown in the curve of Fig. 4, the whole network node voltages are increased significantly, so the program has good effect on improvement of the node voltage and is applicable toincrease of the terminal voltage at the lowpressure side. Drop of the loss in the distribution network is shown in Table 1. With the example simulation of the IEEE33 node distribution system, the drop of the transmission loss is $67.7 \%$. Therefore, in addition to the increase of the terminal voltage, the programachieves the goal of reducing the transmission loss.

Table 1 Position and Capacity of PV into the power grid

\begin{tabular}{|c|c|c|c|}
\hline $\begin{array}{l}\text { Node } \\
\text { number }\end{array}$ & PV Position & $\begin{array}{l}\text { Injection Capacity } \\
(\mathrm{KW})\end{array}$ & Transmission Loss \\
\hline 0 & & 0 & 396.233 \\
\hline 1 & 30 & 800 & 247.684 \\
\hline 2 & 11,30 & 800,800 & 177.606 \\
\hline 3 & $11,24,30$ & $800,1019,800$ & 138.611 \\
\hline 4 & $11,18,24,30$ & $800,752,1019,800$ & 127.887 \\
\hline
\end{tabular}

\section{SPSS Analysis of Parameters ofPV Grid-Connected Node}

\section{A. Analysis of correlation betweenthe PV injection mode and the node voltage}

It uses the node voltage as the defining factor for repeated measurement to conduct the test for five times (no PV injection, node 30 PV injection, nodes 30 and 11 PV injection, nodes 30,11 and 24 PV injection, and nodes 30,11,24 and 18 PV injection) to obtain the condition analysis of node voltage in different PV injection modes. The linear regression analysis is conducted with the node voltage as the dependent variable, and the injection mode and thenode type as independent variables, 
and the $\mathrm{R}$ value is 0.401 in the correlation analysis as shown in Table 2 , showing that the effect on the node voltage of both injection mode and node type is greater than that of the injection modealone, as well as that the selection of PV injection mode is the key reasonable PV grid connection.

Table 2

\begin{tabular}{ccccc}
\multicolumn{4}{c}{ Analysis of Correlation between Node Voltage and PV Injection Mode } \\
\hline Model & $\mathrm{R}$ & $\mathrm{R}^{2}$ & Adjusted $\mathrm{R}^{2}$ & Standard estimated error \\
\hline 1 & $.383^{\mathrm{a}}$ & .146 & .119 & .28778889
\end{tabular}

It established a prediction model of the node voltage and the PV injection mode, where the Rsquare value of the univariate prediction model with the injection mode as the independent variable is 0.119 , and its reliability is higher than that of the multivariate prediction model with both injection mode and node type as the independent variable.Also, the standard coefficient $\beta$ of the injection mode in modelling coefficients as shown in Table 3 is 0.387 andis higher than that of the node type of 0.121 , indicating the effect of the injection mode on the node voltageismuch higher than that of the node type.

Table3

\begin{tabular}{|c|c|c|c|c|c|c|}
\hline \multirow{3}{*}{ Model } & \multirow{2}{*}{\multicolumn{2}{|c|}{$\begin{array}{l}\text { Non-standardized } \\
\text { coefficient }\end{array}$}} & \multirow{2}{*}{\multicolumn{2}{|c|}{ Standard Coefficient }} & \multirow{3}{*}{$\mathrm{t}$} & \multirow{3}{*}{$\operatorname{sig}$} \\
\hline & & & & & & \\
\hline & & B & $\begin{array}{c}\text { Standard } \\
\text { Error }\end{array}$ & $\begin{array}{c}\text { Trial } \\
\text { Version }\end{array}$ & & \\
\hline \multirow[t]{2}{*}{1} & (Constant) & 9.752 & .123 & & 79.295 & .000 \\
\hline & $\begin{array}{l}\text { Injection } \\
\text { Mode }\end{array}$ & .098 & .043 & .383 & 2.306 & .028 \\
\hline \multirow[t]{3}{*}{2} & (Constant) & 9.638 & .200 & & 48.147 & .000 \\
\hline & $\begin{array}{l}\text { Injection } \\
\text { Mode }\end{array}$ & .099 & .043 & .387 & 2.311 & .028 \\
\hline & Node Type & .034 & .046 & .121 & .725 & .474 \\
\hline
\end{tabular}

Therefore, the node type is treated as the removed variable, while only the prediction model with the injection mode as the independent variable is considered.The obtained linear regression equation for the node voltage and the injection mode is ${ }^{y}=0.098 x+9.752$; Durbin-Watson test value is 1.306 , and the error term is independent, so the linear regression analysis is reliable. To test the fit of the prediction model, the residualanalysis was conducted further. The residuals are distributed along the axis, showing that the test sample is normally distributed. The test samples are distributed around the 0axis, and both upper and lower limits are within 3 . This shows that the test sample is in line with the normal distribution, while the variance is homogeneous; therefore, the independence assumption is valid.

In the distribution system, the intermittent nature of photovoltaic power affects the reliability of the grid-connected operation. From the prediction model, we can selectively operate the PV gridconnected system of the whole grid and predict the changes in voltage of the whole grid; on the contrary, we can control the PV grid-connected conditions by the voltage value of the specific nodeaccording to the userdemand at the low pressure side so that we obtain the PV gridconnectedbilateral interactive control strategy.

B. Effect of PV injection mode on the end node voltage

The terminal node of the IEEE33 node distribution system is taken forthe small sample $\mathrm{T}$ paired test; the paired samplecorrelation coefficients are shown in Table 4 . The significance probability $\mathrm{P}$ of the end node voltage of nodes $30,11,24$ and 18 after and before PV injection is $0.000<0.05$, so the difference at the 95\% confidence level is significant, i.e.the voltages of the end node with and without four-node PV injection are significantly correlated. Among the PV injected four nodes,the significance of the inter-node voltage at node18 after PV injection is highest, followed by nodes 11, 30and 24. With correlation and significance analyses, it identified the priority for the fournodes, and proposed reasonable suggestions for distribution network reconstruction. In case of emergencies or circuit failure, you can cut off the low-priority PV grid-connectedsystem and retain the high-priority one. Based on the analysis in view of improvement of the end node voltage in virtue of the PV gridconnection, the node with higher significance has better improvement effect on the end node voltage. 
Table 4

Paired Sample Correlation Coefficients of End Node Voltage with Different PV Injection Modes

\begin{tabular}{clccc}
\hline \multicolumn{1}{c}{ Type } & N & CorrelationCoefficient & Sig \\
\hline Pair1 & $\begin{array}{l}\text { Node voltage without PV injection \&node 30 } \\
\text { PV injection } \\
\text { Pair2 }\end{array}$ & $\begin{array}{l}\text { Node voltage without PV injection \& nodes 30 } \\
\text { and 11 PV injection }\end{array}$ & .958 & .042 \\
Pair3 & $\begin{array}{l}\text { Node voltage without PV injection \& nodes } \\
\text { 30,11 and 24 PV injection } \\
\text { Pode voltage without PV injection \& nodes } \\
\text { P0,11, 24 and 18 PV injection }\end{array}$ & 4 & .979 & .021 \\
\hline
\end{tabular}

\section{Conclusions}

This paper introduces the computer binary search algorithm into the load flow calculation with the objective of minimum transmission loss and by aiming at improving the end voltage, proposes the PV grid-connected addressing and sizing method and also optimize the PV grid-connected configuration. Based on the example simulation verification of the IEEE33 node distribution system, the drop of the transmission loss is $67.7 \%$, and the end node voltage is improved by $7.6 \%$ on average, indicating the program is reasonable and effective.

With the SPSS analysis software, this paper conducted the variance analysis with the node parameter of the IEEE33 node distribution system as the sample, and proposed the linear regression equation on the PV grid-connected node voltage and the PV injection mode. This prediction modelrealized good interaction between thePV systems and the main grid.

\section{Acknowledgement}

This work was supported in National Sci-Tech Support Plan sub-task Low-Carbon Housing Courtyard Micro Grid Function Control System (2012BAJ26B01-01);

Liaoning Provincial Department of Education task Study on the Photovoltaic Grid Connection Optimal Control Strategy based on Feedforward Power Prediction (L2014253);

\section{Reference}

[1]Wang Zhiqun, Zhu Shouzhen, Zhou Shuangxi, et al. Study on Distributed Generation Access Locations and Injection Capacity Constraints [J]. Proceedings of the CSU-EPSA, 2005,17 (1): pp.53-58.

[2]CHEN Shuyong, BAO Hai, WU Chunyang,,et al. Direct Grid-tie Power Control Method for DistributedPhotovoltaicGeneration[J].Proceedings of the CSEE, 2011, 31(10):6-119(in Chinese with English abstract).

[3]Zhou Lindeng, ZengYi, GuoKe, et al. Development of photovoltaic grid-connected system with power quality regulatory function[J]. Power system Protection and Control. 2012, 40(9):137145(in Chinese with English abstract).

[4]Pei Wei, Kong Li, Qi Zhiping.Cooperative voltage regulation strategy in distribution network with high penetration level of PV generations. ACTA ENERGIAE SOLARIS SINICA. 2011,32(11):1629-1635

[5]Ju Fei, Grid voltage Stabilization with Single-Node Centralized PV Power Access[J], East China Electric Power. 2014, 42(4):0742-0747(in Chinese with English abstract).

[6]Chen Quan, Li Lingdong, WangQunjing, et al. SimulatioModel of Photovoltaic Generation GridConnected System and Its Impacts on Voltages Stability in Distribution Grid[J]. TRANSACTIONSOF CHINA ELECTROTECHINCAL SOCIETY. 2013, 28(3):241-247. 